January 2008

\title{
Constraints on Deflation from the Equation of State of Dark Energy
}

\author{
Lauris Baum, Paul H. Frampton and Shinya Matsuzaki \\ Department of Physics and Astronomy, University of North Carolina, \\ Chapel Hill, NC 27599.
}

\begin{abstract}
In cyclic cosmology based on phantom dark energy the requirement that our universe satisfy a CBE-condition (Comes Back Empty) imposes a lower bound on the number $N_{\text {cp }}$ of causal patches which separate just prior to turnaround. This bound depends on the dark energy equation of state $w=p / \rho=-1-\phi$ with $\phi>0$. More accurate measurement of $\phi$ will constrain $N_{\mathrm{cp}}$. The critical density $\rho_{c}$ in the model has a lower bound $\rho_{c} \geq\left(10^{9} \mathrm{GeV}\right)^{4}$ or $\rho_{c} \geq\left(10^{18} \mathrm{GeV}\right)^{4}$ when the smallest bound state has size $10^{-15} \mathrm{~m}$, or $10^{-35} \mathrm{~m}$, respectively.
\end{abstract}




\section{Introduction}

Recently two of the authors have proposed [1-3] a scenario for a cyclic universe based on a dark energy component with constant equation of state satisfying $w=p / \rho=-1-\phi$ where $w<-1$ and hence $\phi>0$. The model involves two key ideas: (i) that the universe deflate just prior to the turnaround from expansion to contraction by disintegrating into a very large number $N_{\text {cp }}$ of causal patches. In the notation of [1], note that $N_{\text {cp }}=1 / f^{3}$; (ii) that the contracting universe be empty, meaning that one causal patch at turnaround must contain no matter or black holes, only dark energy. This is called the CBE condition (Comes Back Empty). Implementation of CBE requires, as we shall explain, a lower bound on $N_{c p}$ which depends on the length scale $L$ characterising the smallest bound system. We shall consider both $L=10^{-15} \mathrm{~m}$ for a nucleon then $L \geq 10^{-35} \mathrm{~m}$ for a PPP (Presently Point Particle) meaning a particle which at present is considered to be pointlike, like a quark or a lepton, but which actually has a characteristic size at least a few orders of magnitude smaller than a nucleon but greater than or equal to the Planck scale.

In the foreseeable future, it is expected that the equation of state of the dark energy $w$, and hence $\phi$, will be measured with higher accuracy by, for example, the Planck Surveyor satellite [4]. What we shall show is that this measurement can, within this model, constrain for a given $w$ the number $N_{\mathrm{cp}}$ of causal patches at turnaround by imposing a lower bound thereon.

The plan of the paper is that in Section 2 we discuss the times at which unbinding, causal disconnection and turnaround occur. In Section 3, the constraints on $N_{\mathrm{cp}}$ from measurement of $\phi$ are derived. Finally, Section 4 is

a discussion. In the Appendices is technical material to supplement the main text. 


\section{Times of unbinding, causal disconnection and turnaround}

In this section we analyze four relationships between cosmic times in the cyclic model expansion era: i) $t_{\text {unbound }}$ (at which a bound system will become unbound due to the large dark energy force with $w<-1$ ); ii) $t_{\text {caus }}$ (at which a previously bound system becomes casually disconnected, meaning that no light signal could exchange before the would-be Big Rip; this is how we estimate $N_{\mathrm{cp}}$ ); iii) $t_{T}$ (time when the turnaround occurs); iv) $t_{\text {rip }}$ (at which a "would-be" big rip takes place), in addition to the present time $t_{0}$.

In the Baum-Frampton (BF) model [1], there are three parameters; $w$ (equation of state of dark energy); $\rho_{C}$ (critical density which the total density in the system $\rho_{\text {tot }}$ reached at $\left.t=t_{T}\right) ; f$ (the deflation fraction parameter related to the number of causal patches by $\left.N_{\text {cp }}=\left(1 / f^{3}\right)\right)$. We will analyze the model taking the value of $w$ lying in a range,

$$
-1.10000 \leq \omega \leq-1.00001
$$

and for $\rho_{C}$ choosing the following range,

$$
\left(10^{3} \mathrm{GeV}\right)^{4} \leq \rho_{C} \leq\left(10^{19} \mathrm{GeV}\right)^{4} .
$$

The choice of the range of $w$ is motivated by the current lower bound from observations $[5,6]$ and the upper bound, by the cosmic variance uncertainty in this measurement.

Reserving details of the derivation of four formulas to Appendix A, we shall here refer to the resultant expressions:

- $\left(t_{\text {rip }}-t_{0}\right)$

$$
t_{\text {rip }}-t_{0} \simeq \frac{11 \mathrm{Gyr}}{|1+w|} .
$$

- $\left(t_{\text {rip }}-t_{\text {unbound }}\right)$

$$
t_{\text {rip }}-t_{\text {unbound }}=\alpha(w) P \text {, }
$$

where [7]

$$
\alpha(w)=\frac{\sqrt{2|1+3 w|}}{6 \pi|1+w|}
$$

and $P$ denotes the period associated with the binding force which had been constraining objects into a certain bound system before $t=$ $t_{\text {unbound }}$. 
- $\left(t_{\text {rip }}-t_{\text {caus }}\right)$

$$
t_{\text {rip }}-t_{\text {caus }}=\left|\frac{1+3 w}{3(1+w)}\right|\left(\frac{L}{c}\right)
$$

where $c$ is the speed of light and $L$ stands for the length scale of the bound system [7].

- $\left(t_{\text {rip }}-t_{T}\right)$

$$
t_{\text {rip }}-t_{T}=\frac{11 \mathrm{Gyr}}{|1+w|} 10^{-14.5} \eta^{-1 / 2}
$$

where $\eta$ is a scale factor of $\rho_{C}$ defined by $\rho_{C}=\eta \rho_{\mathrm{H}_{2} \mathrm{O}}$ with $\rho_{\mathrm{H}_{2} \mathrm{O}}$ being the density if water, $\rho_{H_{2} O}=1 \mathrm{~g} \cdot \mathrm{cm}^{-3}$. Eq.(17) appeared \#1 as Eq.(4) in Ref. [1].

The numerical analysis for these relationships is presented in Appendices $\mathrm{A}$ and $\mathrm{B}$. As a result, we find the lower bound for $\rho_{C}$,

$$
\rho_{C}>\left(10^{18} \mathrm{GeV}\right)^{4}
$$

which is obtained by imposing that the time for a presently point particle (PPP), with the size $10^{-33} \mathrm{~m}=\mathrm{L}$, satisfy $t_{\text {rip }}>t_{T}>t_{\text {caus }}^{\mathrm{PPP}}>t_{\text {unbound }}^{\mathrm{PPP}}$. It should be emphasized that this result is almost independent of a choice of $w$ in the range of interest.

For a nucleon with $L \simeq 10^{-15} \mathrm{~m}$, the corresponding lower bound is

$$
\rho_{C} \gtrsim\left(10^{9} \mathrm{GeV}\right)^{4} .
$$

\footnotetext{
${ }^{\# 1}$ Eq.(77) corrects a typo in the exponent of $\eta$ appearing in [1]
} 


\section{Given $w$, the constraints on $N_{\mathrm{cp}}$}

In [1], as in [7], various bound systems were discussed including galaxies, the Earth-Sun system, the hydrogen atom and a nucleon. Each may be charaterised by a present length scale $L_{0}$.

For the CBE condition we must insist that the smallest bound systems are disintegrated before turnaround which means that the size of a generic causal patch $L_{\mathrm{cp}}$ (to be defined below) is smaller than the size $L\left(t_{T}\right)$ at turnaround of the bound system whose present length scale is $L\left(t_{0}\right)=L_{0}$, namely

$$
L_{\text {cp }} \leq L\left(t_{T}\right)=L_{0}\left(\frac{a\left(t_{T}\right)}{a\left(t_{\text {unbound }}\right)}\right) .
$$

We remind the reader that the CBE condition is mandatory because if the contracting universe contains matter it will not generally contract sufficiently but will undergo a premature bounce. Even if a causal patch contains only one very infra-red photon, this can blue-shift to an energy sufficient to create $e^{+} e^{-}$pairs before the bounce, again disallowing sufficient contraction for infinite cyclicity.

This was the motivation for [3] where it was shown that the mean number of low-energy photons per causal patch is much less than one and is essentially zero. There will always be a vanishing but strictly non-zero number of patches which fail to cycle but it was shown in [2] that the probability of a successful universe is equal to one; it was noted that the total number of universes has always been, and always will be constantly infinite and equal to $\aleph_{0}$ (Alephzero). $\aleph_{0}$ is a countable infinity, exemplified by the number of primes, of integers or of rational numbers.

To enable infinite cyclicity we must have the CBE condition, Eq.(10), for the smallest bound systems. The smallest bound systems we know about are nucleons with $L_{0}=10^{-15} \mathrm{~m}$.

To be general, we consider PPPs (Presently Point Particles) meaning particles which are presently regarded as pointlike but may not be. We allow a bound state scale for PPPs to be anywhere between the present upper limit of about $(1 \mathrm{TeV})^{-1}=10^{-18} \mathrm{~m}$ and the Planck scale of $10^{-35} \mathrm{~m}$. As we shall see shortly, the lower bound on $N_{\text {cp }}$ is so sensitive to where $L_{0}$ is chosen within these twenty orders of magnitude that its presentation requires us to plot

$\log _{10} \log _{10} N_{\text {cp }}$ against the equation of state of the dark energy. 


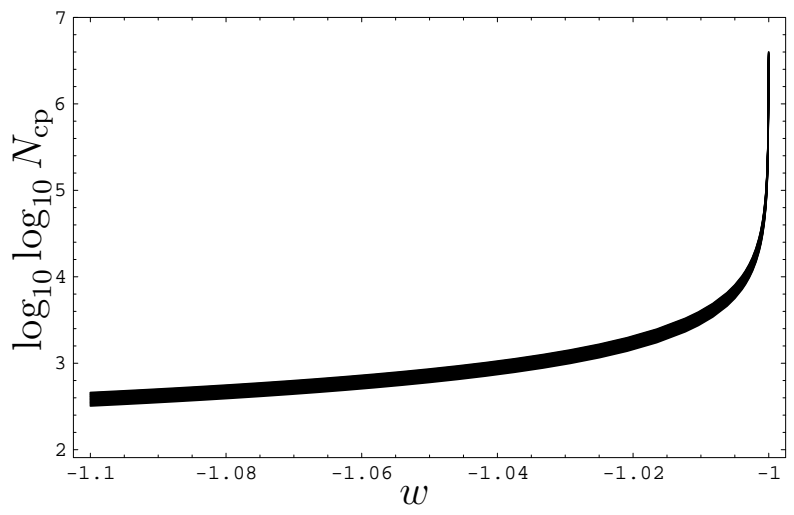

Figure 1: Constraint on $w$ and $N_{\mathrm{cp}}$ coming from the CBE condition (Comes Back Empty), corresponding to inequality (13). The black band in this figure has been created by varying the length of smaller bound systems from $L_{p}\left(t_{0}\right)=10^{-33} \mathrm{~m}$ to $L_{p}\left(t_{0}\right)=10^{-15} \mathrm{~m}$; the bottom edge corresponds to the lower value of $L_{p}\left(t_{0}\right)$. The region below the black band is forbidden by the CBE condition.

The present Hubble length $r_{H}\left(t_{0}\right)$ is given by

$$
r_{H}\left(t_{0}\right)=\frac{1}{H_{0}}
$$

which, at the turnaround, would naively become

$$
r_{H}\left(t_{T}\right)=r_{H}\left(t_{0}\right) a\left(t_{T}\right)
$$

since by definition $a\left(t_{0}\right)=1$.

In the cyclic model of [1], the size of a causal patch $L_{\mathrm{cp}}$ is instead defined by

$$
L_{\mathrm{cp}}=\frac{r_{H}\left(t_{T}\right)}{N_{\mathrm{cp}}}
$$

and therefore Eq.(10) can be calculated for different values of $L_{0}$, see Appendix C. The results are illustrated in Figure 1 where we plot $\log _{10} \log _{10} N_{\text {cp }}$ versus $w=-1-\phi$.

From this figure we see that a measurement of $w$ in the range anticipated for the Planck surveyor will provide a lower bound on $N_{\text {cp }}$. For example $w=-1.05$ implies $N_{\mathrm{cp}} \gtrsim 10^{630}$ for disintegration of nucleons and $N_{\mathrm{cp}} \gtrsim 10^{1000}$ for disintegration of PPPs with bound scale at the Planck length. 


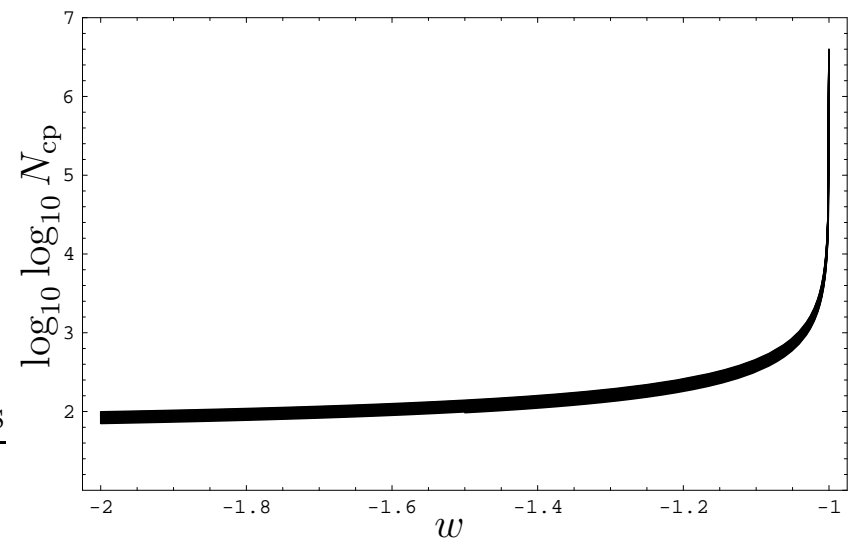

Figure 2: Constraint for $-2 \leq w \leq-1.1$ and $N_{\mathrm{cp}}$ coming from the CBE condition (Comes Back Empty), corresponding to inequality (13). The black band in this figure has been created by varying the length of smaller bound systems from $L_{p}\left(t_{0}\right)=10^{-33} \mathrm{~m}$ to $L_{p}\left(t_{0}\right)=10^{-15} \mathrm{~m}$; the bottom edge corresponds to the lower value of $L_{p}\left(t_{0}\right)$. The region below the black band is forbidden by the CBE condition.

Since we know the entropy of the present universe is at least $S\left(t_{0}\right) \gtrsim 10^{102}$ $[8,9]$ one must impose

$$
N_{\mathrm{cp}}>10^{102}
$$

and, by requiring only the dissociation of nucleons we see from Figure 2 that this implies

$$
w \gtrsim-2
$$

Of course, WMAP data [6] already guarantee this condition but it is interesting that the cyclic model of [1] would be impossible if Eq.(15) had been violated. 


\section{Discussion}

What we have deduced is that the parameters $\rho_{c}, w$ and $N_{\text {cp }}$ in cyclic cosmology are already constrained by existing data. For example one requires $w \gtrsim-2$ for the CBE aspect to work.

This constraint is already known to be respected in Nature but as better and more accurate cosmological data become available it will shed further light on the viability of the theory.

In particular, the accurate measurement of the equation of state $w=$ $-1-\phi$ is of special interest. Fortunately the Planck Surveyor [4] is anticipated to acquire improved accuracy on $w$ in the near future. As we have discussed, this will provide a lower bound on the number $N_{\mathrm{cp}}$ of causal patches necessary to dissociate the smallest bound systems at turnaround and hence to solve the entropy problem and, via CBE, enable the possibility of infinite cyclicity.

It is amusing that the physical conditions at the approach of deflation are so extraordinary that it is natural to ask whether the systems presently regarded as point particles may be composite because the phantom dark energy density grows to unimaginably large values and can disintegrate bound systems down to arbitrarily small scales. We have conservatively limited our attention to systems bigger than the Planck length. However, although this requirement seems dictated by considerations of quantum gravity, it is possible that the dark energy will dissociate even smaller systems if they exist.

The advantage of cyclic cosmology is that it removes the initial singularity associated with the Big Bang, about 13.7 billion years ago, and allows that time never began. The previous attempts to create a consistent infinite cyclicity were stymied between about 1934 [10] and 2002 [11] primarily because of the entropy problem and the second law of thermodynamics. The discovery of the accelerated expansion rate of the universe and the concomitant necessity of dark energy has permitted more optimism that the cyclic cosmology is, after all, on the right track. 


\section{Acknowledgments}

This work was supported in part by the U.S. Department of Energy under Grant No. DG-FG02-06ER41418. 


\section{Appendix A}

\section{Derivation of formulas and numerical analysis}

We begin by writing down the Friedman equation for times $\left(t_{0}<t<t_{T}\right.$ which correspond to the expansion phase [1],

$$
H^{2}(t) \equiv\left(\frac{\dot{a}(t)}{a(t)}\right)^{2}=\frac{8 \pi G}{3}\left[\frac{\rho_{\Lambda}^{0}}{[a(t)]^{3(1+w)}}-\frac{\left[\rho_{\mathrm{tot}}(t)\right]^{2}}{\rho_{C}}\right],
$$

where we have put $\Omega_{r}^{0}=\Omega_{m}^{0}=0$. Taking into account the rapid acceleration when $t_{0}<t<t_{T}$ (or $t_{\text {rip }}$ ), we may neglect the last term proportional to $\rho_{\text {tot }}^{2} \sim\left[a^{3(1+w)}\right]^{2}$, so that

$$
\left(\frac{\dot{a}(t)}{a(t)}\right)^{2} \simeq H_{0}^{2} \frac{\Omega_{\Lambda}^{0}}{[a(t)]^{3(1+w)}} .
$$

With the boundary condition $a\left(t_{\text {rip }}\right)=\infty$, by employing the equation of state $w<-1$, Eq.(17) can readily be solved for an arbitrary time $t$ satisfying $t<t_{\text {rip }}$ to get

$$
t_{\text {rip }}-t=\left(H_{0} \sqrt{\Omega_{\Lambda}^{0}}\right)^{-1} \frac{2}{3|1+w|} a(t)^{-\frac{3|1+w|}{2}} .
$$

\section{A1. The formula for $\left(t_{\text {rip }}-t_{0}\right)$}

Taking $t=t_{0}$ at which point $a\left(t_{0}\right)=1$ and using current observational values [5], $H_{0}=73 \mathrm{~km} \cdot \mathrm{s}^{-1} \cdot \mathrm{Mpc}^{-1}$ and $\Omega_{\Lambda}^{0}=0.76$, we find the time interval $\left(t_{\text {rip }}-t_{0}\right)$ from Eq.(18) to be [7]

$$
t_{\text {rip }}-t_{0} \simeq \frac{11 \mathrm{Gyr}}{|1+w|} .
$$

In Table 1 we list the values of $\left(t_{\text {rip }}-t_{0}\right)$ for 37 specific choices of $w$ in the range $-1.10000 \leq \omega \leq-1.00001$. 


\begin{tabular}{r|r}
\hline$w$ & $\left(t_{\text {rip }}-t_{0}\right)[\mathrm{Gyr}]$ \\
\hline \hline-1.10000 & 110 \\
-1.09000 & 122 \\
-1.08000 & 137 \\
-1.07000 & 157 \\
-1.06000 & 183 \\
-1.05000 & 220 \\
-1.04000 & 275 \\
-1.03000 & 366 \\
-1.02000 & 550 \\
-1.01000 & 1100 \\
-1.00900 & 1222 \\
-1.00800 & 1375 \\
-1.00700 & 1571 \\
-1.00600 & 1833 \\
-1.00500 & 2200 \\
-1.00400 & 2750 \\
-1.00300 & 3666 \\
-1.00200 & 5500 \\
-1.00100 & 11000 \\
-1.00090 & 12222 \\
-1.00080 & 13750 \\
-1.00070 & 15714 \\
-1.00060 & 18333 \\
-1.00050 & 22000 \\
-1.00040 & 27500 \\
-1.00030 & 36666 \\
-1.00020 & 55000 \\
-1.00010 & 110000 \\
-1.00009 & 122222 \\
-1.00008 & 137500 \\
-1.00007 & 157143 \\
-1.00006 & 183333 \\
-1.00005 & 220000 \\
-1.000000 & 275000 \\
\hline & \\
\hline
\end{tabular}

Table 1: Values of $\left(t_{\text {rip }}-t_{0}\right)$ for $-1.10000 \leq \omega \leq-1.00001$. 


\section{A2. The formula for $\left(t_{\text {rip }}-t_{T}\right)$}

Putting $t=t_{T}$ in Eq.(18) and dividing both sides by $\left(t_{\text {rip }}-t_{0}\right)$, we find a relationship independent of both $H_{0}$ and $\Omega_{\Lambda}^{0}$,

$$
\frac{t_{\text {rip }}-t_{T}}{t_{\text {rip }}-t_{0}}=\left[a\left(t_{T}\right)\right]^{-\frac{3 \phi}{2}} .
$$

We shall recall here that the turnaround-time $t_{T}$ is characterized by $\rho_{\Lambda}\left(t_{T}\right)=$

$\rho_{C}$, derived from examining a solution $H^{2}=0$ of Eq.(16), which allows us to rewrite Eq.(20) as

$$
\frac{t_{\text {rip }}-t_{T}}{t_{\text {rip }}-t_{0}}=\sqrt{\frac{\rho_{\Lambda}^{0}}{\rho_{C}}},
$$

where we have calculated the right-hand side using

$$
\left[a\left(t_{T}\right)\right]^{-3 \phi}=\frac{\rho_{\Lambda}\left(t_{0}\right)}{\rho_{\Lambda}\left(t_{T}\right)}=\frac{\rho_{\Lambda}\left(t_{0}\right)}{\rho_{C}} .
$$

Following Ref. [1], we may introduce a unit of energy density, $\rho_{\mathrm{H}_{2} \mathrm{O}}$, in such a way that the critical density $\rho_{C}$ is scaled by a factor of $\eta$

$$
\rho_{C} \equiv \eta \cdot \rho_{H_{2} O}
$$

The present dark energy density $\rho_{\Lambda}^{0}$ can be expressed in terms of $\rho_{\mathrm{H}_{2} \mathrm{O}}=$ $1 \mathrm{~g} / \mathrm{cm}^{3}$ as

$$
\rho_{\Lambda}^{0}=10^{-29} \rho_{\mathrm{H}_{2} \mathrm{O}}
$$

which immediately leads us to

$$
\frac{\rho_{\Lambda}^{0}}{\rho_{C}}=\frac{10^{-29} \rho_{H_{2} O}}{\eta \cdot \rho_{H_{2} O}}=\eta^{-1} 10^{-29} .
$$

Hence we have [1]

$$
t_{\text {rip }}-t_{T}=\left(t_{\text {rip }}-t_{0}\right) \cdot 10^{-14.5} \cdot \eta^{-1 / 2}
$$

In Tables 2 to 4 , choosing $\eta=10^{29}, 10^{57}, 10^{93}$, respectively, corresponding to $\rho_{C} \simeq\left(10^{3} \mathrm{GeV}\right)^{4},\left(10^{10} \mathrm{GeV}\right)^{4},\left(10^{19} \mathrm{GeV}\right)^{4}$ in units of $\rho_{H_{2} O}$, we list the values for the time interval $\left(t_{\text {rip }}-t_{T}\right)$, which turn out to be at most of order $\mathcal{O}\left(10^{-7} \mathrm{~s}\right)$. 


\begin{tabular}{|c|c|c|}
\hline \multicolumn{3}{|c|}{$\eta=10^{29}$} \\
\hline$w$ & $\left(t_{\text {rip }}-t_{T}\right)[$ & \\
\hline 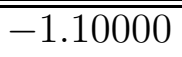 & & $3.4 \times 10^{-11}$ \\
\hline-1.09000 & & $3.8 \times 10^{-11}$ \\
\hline-1.08000 & & $4.3 \times 10^{-11}$ \\
\hline-1.07000 & & $4.9 \times 10^{-11}$ \\
\hline-1.06000 & & $5.7 \times 10^{-11}$ \\
\hline-1.05000 & & $6.9 \times 10^{-11}$ \\
\hline-1.04000 & & $8.6 \times 10^{-11}$ \\
\hline-1.03000 & & $1.1 \times 10^{-10}$ \\
\hline-1.02000 & & $1.7 \times 10^{-10}$ \\
\hline-1.01000 & & $3.4 \times 10^{-10}$ \\
\hline-1.00900 & & $3.8 \times 10^{-10}$ \\
\hline-1.00800 & & $4.3 \times 10^{-10}$ \\
\hline-1.00700 & & $4.9 \times 10^{-10}$ \\
\hline-1.00600 & & $5.7 \times 10^{-10}$ \\
\hline-1.00500 & & $6.9 \times 10^{-10}$ \\
\hline-1.00400 & & $8.6 \times 10^{-10}$ \\
\hline-1.00300 & & $1.1 \times 10^{-9}$ \\
\hline-1.00200 & & $1.7 \times 10^{-9}$ \\
\hline-1.00100 & & $3.4 \times 10^{-9}$ \\
\hline-1.00090 & & $3.8 \times 10^{-9}$ \\
\hline-1.00080 & & $4.3 \times 10^{-9}$ \\
\hline-1.00070 & & $4.9 \times 10^{-9}$ \\
\hline-1.00060 & & $5.7 \times 10^{-9}$ \\
\hline-1.00050 & & $6.9 \times 10^{-9}$ \\
\hline-1.00040 & & $8.6 \times 10^{-9}$ \\
\hline-1.00030 & & $1.1 \times 10^{-8}$ \\
\hline-1.00020 & & $1.7 \times 10^{-8}$ \\
\hline-1.00010 & & $3.4 \times 10^{-8}$ \\
\hline-1.00009 & & $3.8 \times 10^{-8}$ \\
\hline-1.00008 & & $4.3 \times 10^{-8}$ \\
\hline-1.00007 & & $4.9 \times 10^{-8}$ \\
\hline-1.00006 & & $5.7 \times 10^{-8}$ \\
\hline-1.00005 & & $6.9 \times 10^{-8}$ \\
\hline-1.00004 & & $8.6 \times 10^{-8}$ \\
\hline-1.00003 & & $1.1 \times 10^{-7}$ \\
\hline-1.00002 & & $1.7 \times 10^{-7}$ \\
\hline-1.00001 & & $3.4 \times 10^{-7}$ \\
\hline
\end{tabular}

Table 2: Values of $\left(t_{\text {rip }}-t_{T}\right)$ for $-1.10000 \leq \omega \leq-1.00001$ with $\eta=10^{29}$ fixed. 


\begin{tabular}{|c|c|c|}
\hline \multicolumn{3}{|c|}{$\eta=10^{57}$} \\
\hline$w$ & $\left(t_{\text {rip }}-t_{T}\right)[$ & \\
\hline "-1.10000 & & $3.4 \times 10^{-25}$ \\
\hline-1.09000 & & $3.8 \times 10^{-25}$ \\
\hline-1.08000 & & $4.3 \times 10^{-25}$ \\
\hline-1.07000 & & $4.9 \times 10^{-25}$ \\
\hline-1.06000 & & $5.7 \times 10^{-25}$ \\
\hline-1.05000 & & $6.9 \times 10^{-25}$ \\
\hline-1.04000 & & $8.6 \times 10^{-25}$ \\
\hline-1.03000 & & $1.1 \times 10^{-24}$ \\
\hline-1.02000 & & $1.7 \times 10^{-24}$ \\
\hline-1.01000 & & $3.4 \times 10^{-24}$ \\
\hline-1.00900 & & $3.8 \times 10^{-24}$ \\
\hline-1.00800 & & $4.3 \times 10^{-24}$ \\
\hline-1.00700 & & $4.9 \times 10^{-24}$ \\
\hline-1.00600 & & $5.7 \times 10^{-24}$ \\
\hline-1.00500 & & $6.9 \times 10^{-24}$ \\
\hline-1.00400 & & $8.6 \times 10^{-24}$ \\
\hline-1.00300 & & $1.1 \times 10^{-23}$ \\
\hline-1.00200 & & $1.7 \times 10^{-23}$ \\
\hline-1.00100 & & $3.4 \times 10^{-23}$ \\
\hline-1.00090 & & $3.8 \times 10^{-23}$ \\
\hline-1.00080 & & $4.3 \times 10^{-23}$ \\
\hline-1.00070 & & $4.9 \times 10^{-23}$ \\
\hline-1.00060 & & $5.7 \times 10^{-23}$ \\
\hline-1.00050 & & $6.9 \times 10^{-23}$ \\
\hline-1.00040 & & $8.6 \times 10^{-23}$ \\
\hline-1.00030 & & $1.1 \times 10^{-22}$ \\
\hline-1.00020 & & $1.7 \times 10^{-22}$ \\
\hline-1.00010 & & $3.4 \times 10^{-22}$ \\
\hline-1.00009 & & $3.8 \times 10^{-22}$ \\
\hline-1.00008 & & $4.3 \times 10^{-22}$ \\
\hline-1.00007 & & $4.9 \times 10^{-22}$ \\
\hline-1.00006 & & $5.7 \times 10^{-22}$ \\
\hline-1.00005 & & $6.9 \times 10^{-22}$ \\
\hline-1.00004 & & $8.6 \times 10^{-22}$ \\
\hline-1.00003 & & $1.1 \times 10^{-21}$ \\
\hline-1.00002 & & $1.7 \times 10^{-21}$ \\
\hline-1.00001 & & $3.4 \times 10^{-21}$ \\
\hline
\end{tabular}

Table 3: Values of $\left(t_{\text {rip }}-t_{T}\right)$ for $-1.10000 \leq \omega \leq-1.00001$ with $\eta=10^{57}$ fixed. 


\begin{tabular}{|c|c|c|}
\hline \multicolumn{3}{|c|}{$\eta=10^{93}$} \\
\hline$w$ & $\left(t_{\text {rip }}-t_{T}\right)[$ & \\
\hline "-1.10000 & & $3.4 \times 10^{-43}$ \\
\hline-1.09000 & & $3.8 \times 10^{-43}$ \\
\hline-1.08000 & & $4.3 \times 10^{-43}$ \\
\hline-1.07000 & & $4.9 \times 10^{-43}$ \\
\hline-1.06000 & & $5.7 \times 10^{-43}$ \\
\hline-1.05000 & & $6.9 \times 10^{-43}$ \\
\hline-1.04000 & & $8.6 \times 10^{-43}$ \\
\hline-1.03000 & & $1.1 \times 10^{-42}$ \\
\hline-1.02000 & & $1.7 \times 10^{-42}$ \\
\hline-1.01000 & & $3.4 \times 10^{-42}$ \\
\hline-1.00900 & & $3.8 \times 10^{-42}$ \\
\hline-1.00800 & & $4.3 \times 10^{-42}$ \\
\hline-1.00700 & & $4.9 \times 10^{-42}$ \\
\hline-1.00600 & & $5.7 \times 10^{-42}$ \\
\hline-1.00500 & & $6.9 \times 10^{-42}$ \\
\hline-1.00400 & & $8.6 \times 10^{-42}$ \\
\hline-1.00300 & & $1.1 \times 10^{-41}$ \\
\hline-1.00200 & & $1.7 \times 10^{-41}$ \\
\hline-1.00100 & & $3.4 \times 10^{-41}$ \\
\hline-1.00090 & & $3.8 \times 10^{-41}$ \\
\hline-1.00080 & & $4.3 \times 10^{-41}$ \\
\hline-1.00070 & & $4.9 \times 10^{-41}$ \\
\hline-1.00060 & & $5.7 \times 10^{-41}$ \\
\hline-1.00050 & & $6.9 \times 10^{-41}$ \\
\hline-1.00040 & & $8.6 \times 10^{-41}$ \\
\hline-1.00030 & & $1.1 \times 10^{-40}$ \\
\hline-1.00020 & & $1.7 \times 10^{-40}$ \\
\hline-1.00010 & & $3.4 \times 10^{-40}$ \\
\hline-1.00009 & & $3.8 \times 10^{-40}$ \\
\hline-1.00008 & & $4.3 \times 10^{-40}$ \\
\hline-1.00007 & & $4.9 \times 10^{-40}$ \\
\hline-1.00006 & & $5.7 \times 10^{-40}$ \\
\hline-1.00005 & & $6.9 \times 10^{-40}$ \\
\hline-1.00004 & & $8.6 \times 10^{-40}$ \\
\hline-1.00003 & & $1.1 \times 10^{-39}$ \\
\hline-1.00002 & & $1.7 \times 10^{-39}$ \\
\hline-1.00001 & & $3.4 \times 10^{-39}$ \\
\hline
\end{tabular}

Table 4: Values of $\left(t_{\text {rip }}-t_{T}\right)$ for $-1.10000 \leq \omega \leq-1.00001$ with $\eta=10^{93}$ fixed. 


\section{A3. The formula for $\left(t_{\text {rip }}-t_{\text {unbound }}\right)$}

Let us next consider a time $t_{\text {unbound }}$ at which point a gravitationally bound system will become unbound due to an extraordinarily rapid expansion of the universe. Roughly speaking, a bound system in circular orbit at radius $R$ with mass $M$ becomes unbound when

$$
\frac{4 \pi}{3} R^{3} \rho_{\Lambda}\left(t_{\text {unbound }}\right)|1+3 w| \simeq M
$$

where the left-hand side comes from the $T_{\mu \nu}$-term in the right-hand side of the Einstein equation.

Putting $t=t_{\text {unbound }}$ in Eq.(18) and rewriting the overall factor $\left(H_{0} \sqrt{\Omega}\right)^{-1}$, in terms of $\rho_{\Lambda}^{0}$ and the gravitational constant $G$, as $\left(H_{0} \sqrt{\Omega}\right)^{-1}=(8 \pi G / 3$. $\left.\rho_{\Lambda}^{0}\right)^{-1 / 2}$, we may express a time interval $\left(t_{\text {rip }}-t_{\text {unbound }}\right)$ as

$$
\begin{aligned}
\left(t_{\text {rip }}-t_{\text {unbound }}\right) & =\left(8 \pi G / 3 \rho_{\Lambda}^{0}\right)^{-1 / 2} \frac{2}{3|1+w|}\left[a\left(t_{\text {unbound }}\right)\right]^{-\frac{3|1+w|}{2}} \\
& =(8 \pi G / 3)^{-1 / 2} \sqrt{\frac{1}{\rho_{\Lambda}^{0}} \frac{2}{3|1+w|} \sqrt{\frac{\rho_{\Lambda}^{0}}{\rho_{\Lambda}\left(t_{\text {unbound }}\right)}}} \\
& =(8 \pi G / 3)^{-1 / 2} \frac{2}{3|1+w|} \sqrt{\frac{1}{\rho_{\Lambda}\left(t_{\text {unbound }}\right)}} .
\end{aligned}
$$

Using Eq.(27) we can further rewrite the right-hand side as

$$
\begin{aligned}
\left(t_{\text {rip }}-t_{\text {unbound }}\right) & =\frac{\sqrt{2|1+3 w|}}{3|1+w|} \sqrt{\frac{R^{3}}{G M}} \\
& =\frac{\sqrt{2|1+3 w|}}{6 \pi|1+w|} P,
\end{aligned}
$$

where in the last line we have used a relationship from classical gravitational systems,

$$
P=2 \pi \sqrt{\frac{R^{3}}{G M}},
$$

in which $P$ denotes the period for a circular orbit of radius $R$ around a system bound by gravitational force with mass $M$. Thus we reach the expression [7] for the time interval $\left(t_{\text {rip }}-t_{\text {unbound }}\right)$,

$$
\left(t_{\text {rip }}-t_{\text {unbound }}\right)=\alpha(w) P,
$$

where

$$
\alpha(w)=\frac{\sqrt{2|1+3 w|}}{6 \pi|1+w|} .
$$


Similarly to Eq.(27), even for binding forces other than gravity, we can roughly estimate an unbound-time $t_{\text {unbound. }}$. For simplicity, we shall derive here a relationship similar to Eq.(27) focusing on an electromagnetically bound system, e.g., a hydrogen atom $H$, in which the electron is constrained on a circular orbit of radius $R$ by the Coulomb force around a proton. This can be done just by taking into account the balance problem between the Coulomb force $F_{C}$ and the dark energy force $F_{w}$. We find that the system will become unbound when

$$
\begin{aligned}
F_{C} & \simeq F_{w}\left(t_{\text {unbound }}\right), \\
\rightarrow \quad \frac{e^{2}}{4 \pi \epsilon_{0}} \frac{1}{R^{2}} & \simeq G \frac{m_{e} m_{\mathrm{eff}}\left(w, t_{\text {unbound }}\right)}{R^{2}},
\end{aligned}
$$

where $m_{\mathrm{eff}}\left(w, t_{\text {unbound }}\right)$ denotes an effective "mass" arising from the dark energy density at $t=t_{\text {unbound }}$,

$$
m_{\text {eff }}\left(w, t_{\text {unbound }}\right)=\frac{4 \pi R^{3}}{3} \rho_{\Lambda}\left(t_{\text {unbound }}\right)|1+3 w| .
$$

Using the expression for the period associated with the electromagnetic force,

$$
P_{\mathrm{em}}=2 \pi \sqrt{\frac{m_{e} R^{3}}{\hbar c \alpha}},
$$

we can rewrite Eq. (33) as

$$
\rho_{\Lambda}\left(t_{\text {unbound }}\right)|1+3 w| \simeq \frac{3 \pi}{G P_{\mathrm{em}}^{2}},
$$

which leads immediately to the formula for $\left(t_{\text {rip }}-t_{\text {unbound }}^{\mathrm{H}}\right)$ in the case of an $\mathrm{H}$ atom. As a result, we find it take the same form as Eq.(31),

$$
t_{\text {rip }}-t_{\text {unbound }}^{\mathrm{H}}=\alpha(w) P_{\mathrm{em}} .
$$

It is straightforward to show that, for other binding forces (e.g. strong forces, etc.), the form of Eq.(37) is unchanged except for replacing $P_{\mathrm{em}}$ with the appropriate one associated with the binding force.

Choosing typical bound systems - galaxy, Sun-Earth, and hydrogen atom - and supplying the corresponding values for the period $P\left(P_{\mathrm{em}}\right)$

\begin{tabular}{c|r|r}
\hline Bound System & \multicolumn{1}{|c|}{$P$} & $L$ \\
\hline \hline Typical Galaxy & $2.0 \times 10 \mathrm{yr}$ & $1.6 \times 10^{4} \mathrm{pc}$ \\
Sun-Earth & $1 \mathrm{yr}$ & $1.5 \times 10^{8} \mathrm{~km}$ \\
Hydrogen Atom & $10^{-16} \mathrm{~s}$ & $0.5 \times 10^{-10} \mathrm{~m}$ \\
\hline
\end{tabular}

we calculate the values of $\left(t_{\text {rip }}-t_{\text {unbound }}\right)$ for each bound system by taking values of $w$ from the range $-1.10000 \leq \omega \leq-1.00001$. The result is summarized in Table 5 . 


\begin{tabular}{|c|c|c|c|}
\hline \multicolumn{4}{|c|}{$\left(t_{\text {rip }}-t_{\text {unbound }}\right)$} \\
\hline$w$ & Typical Galaxy [Gyr] & Sun-Earth [yr] & Hydrogen Atom $[\mathrm{s}]$ \\
\hline -1.10000 & 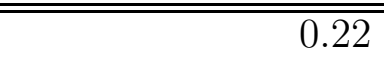 & $\overline{\overline{1.13}}$ & $\overline{1.13 \times 10^{-16}}$ \\
\hline-1.09000 & 0.25 & 1.25 & $1.25 \times 10^{-16}$ \\
\hline-1.08000 & 0.28 & 1.40 & $1.40 \times 10^{-16}$ \\
\hline-1.07000 & 0.31 & 1.59 & $1.59 \times 10^{-16}$ \\
\hline-1.06000 & 0.36 & 1.84 & $1.84 \times 10^{-16}$ \\
\hline-1.05000 & 0.44 & 2.20 & $2.20 \times 10^{-16}$ \\
\hline-1.04000 & 0.54 & 2.73 & $2.73 \times 10^{-16}$ \\
\hline-1.03000 & 0.72 & 3.61 & $3.61 \times 10^{-16}$ \\
\hline-1.02000 & 1.07 & 5.38 & $5.38 \times 10^{-16}$ \\
\hline-1.01000 & 2.13 & 10.6 & $1.06 \times 10^{-15}$ \\
\hline-1.00900 & 2.37 & 11.8 & $1.18 \times 10^{-15}$ \\
\hline-1.00800 & 2.66 & 13.3 & $1.33 \times 10^{-15}$ \\
\hline-1.00700 & 3.04 & 15.2 & $1.52 \times 10^{-15}$ \\
\hline-1.00600 & 3.55 & 17.7 & $1.77 \times 10^{-15}$ \\
\hline-1.00500 & 4.26 & 21.3 & $2.13 \times 10^{-15}$ \\
\hline-1.00400 & 5.32 & 26.6 & $2.66 \times 10^{-15}$ \\
\hline-1.00300 & 7.08 & 35.4 & $3.54 \times 10^{-15}$ \\
\hline-1.00200 & 10.2 & 53.1 & $5.31 \times 10^{-15}$ \\
\hline-1.00100 & 21.2 & 106 & $1.06 \times 10^{-14}$ \\
\hline-1.00090 & 23.5 & 117 & $1.17 \times 10^{-14}$ \\
\hline-1.00080 & 26.5 & 132 & $1.32 \times 10^{-14}$ \\
\hline-1.00070 & 30.3 & 151 & $1.51 \times 10^{-14}$ \\
\hline-1.00060 & 35.3 & 176 & $1.76 \times 10^{-14}$ \\
\hline-1.00050 & 42.4 & 212 & $2.12 \times 10^{-14}$ \\
\hline-1.00040 & 53.0 & 265 & $2.65 \times 10^{-14}$ \\
\hline-1.00030 & 70.7 & 353 & $3.53 \times 10^{-14}$ \\
\hline-1.00020 & 106 & 530 & $5.30 \times 10^{-14}$ \\
\hline-1.00010 & 212 & 1061 & $1.06 \times 10^{-13}$ \\
\hline-1.00009 & 235 & 1179 & $1.17 \times 10^{-13}$ \\
\hline-1.00008 & 265 & 1326 & $1.32 \times 10^{-13}$ \\
\hline-1.00007 & 303 & 1515 & $1.51 \times 10^{-13}$ \\
\hline-1.00006 & 353 & 1768 & $1.76 \times 10^{-13}$ \\
\hline-1.00005 & 424 & 2122 & $2.12 \times 10^{-13}$ \\
\hline-1.00004 & 530 & 2652 & $2.65 \times 10^{-13}$ \\
\hline-1.00003 & 707 & 3536 & $3.53 \times 10^{-13}$ \\
\hline-1.00002 & 1060 & 5305 & $5.30 \times 10^{-13}$ \\
\hline-1.00001 & 2120 & 10610 & $1.06 \times 10^{-12}$ \\
\hline
\end{tabular}

Table 5: Values of $\left(t_{\text {rip }}-t_{\text {unbound }}\right)$ for $-1.10000 \leq \omega \leq-1.00001$ 


\section{A4. The formula for $\left(t_{\text {rip }}-t_{\text {caus }}\right)$}

After $t=t_{\text {unbound }}$, objects which had been constrained in a bound system would be free to move far apart and will end up causally disconnected starting at time $t=t_{\text {caus }}$. Such a time, $t_{\text {caus }}$, can be defined, with $c=1$ taken, by

$$
\frac{L}{a\left(t_{\text {caus }}\right)}=\int_{t_{\text {caus }}}^{t_{\text {rip }}} \frac{d t}{a(t)},
$$

where $L$ denotes the length scale at which two objects separate at $t=t_{\text {caus }}$, and the right-hand side stands for the comoving distance of light which arises from traveling at light speed $c$ during a time-interval $t_{\text {caus }}<t<t_{\text {rip. }}$. Noting that $d t=d a /(a H)$ and rewriting $H(a)$ from the Friedman equation in terms of a function of $a$, we calculate more explicitly the right-hand side of Eq.(38) as follows:

$$
\begin{aligned}
\frac{L}{a\left(t_{\text {caus }}\right)} & =\int_{a\left(t_{\text {caus }}\right)}^{a\left(t_{\text {rip }}\right)} \frac{d a}{a^{2} H(a)} \\
& =\left(H_{0} \cdot \Omega_{\Lambda}^{0}\right)^{-1} \int_{a\left(t_{\text {caus }}\right)}^{\infty} d a a^{-1 / 2(1-3 w)} \\
& =\left(H_{0} \Omega_{\Lambda}^{0}\right)^{-1} \frac{2}{|1+3 w|}\left[a\left(t_{\text {caus }}\right)\right]^{-|1+3 w| / 2}
\end{aligned}
$$

Taking $t=t_{\text {caus }}$ in Eq.(18) and dividing both sides by the resultant expression, we can continue calculating to get

$$
\begin{aligned}
\frac{L}{a\left(t_{\text {caus }}\right)} \frac{1}{\left(t_{\text {rip }}-t_{\text {caus }}\right)} & =\frac{3|1+w|}{|1+3 w|}\left(\frac{\left[a\left(t_{\text {caus }}\right)\right]^{3|1+w|}}{\left[a\left(t_{\text {caus }}\right)\right]^{|1+3 w|}}\right)^{1 / 2} \\
& =\frac{3|1+w|}{|1+3 w|} \frac{1}{a\left(t_{\text {caus }}\right)}
\end{aligned}
$$

and in the end we reach the expression [7]

$$
t_{\text {rip }}-t_{\text {caus }}=\left|\frac{1+3 w}{3(1+w)}\right| \frac{L}{c} .
$$

Similarly to the previous section, we take values of $w$ in the range $-1.10000 \leq$ $\omega \leq-1.00001$ and calculate the values of $\left(t_{\text {rip }}-t_{\text {caus }}\right)$ for typical bound systems such as galaxies, Sun-Earth, hydrogen atom, and summarize in Table 6 . 


\begin{tabular}{|c|c|c|c|}
\hline \multicolumn{4}{|c|}{$\left(t_{\text {rip }}-t_{\text {caus }}\right)$} \\
\hline$w$ & Typical Galaxy [Myr] & Sun-Earth [day] & Hydrogen Atom [s] \\
\hline-1.10000 & 0.40 & 0.044 & $1.27 \times 10^{-18}$ \\
\hline-1.09000 & 0.44 & 0.048 & $1.40 \times 10^{-18}$ \\
\hline-1.08000 & 0.49 & 0.054 & $1.55 \times 10^{-18}$ \\
\hline-1.07000 & 0.55 & 0.060 & $1.75 \times 10^{-18}$ \\
\hline-1.06000 & 0.64 & 0.070 & $2.01 \times 10^{-18}$ \\
\hline-1.05000 & 0.75 & 0.083 & $2.39 \times 10^{-18}$ \\
\hline-1.04000 & 0.93 & 0.102 & $2.94 \times 10^{-18}$ \\
\hline-1.03000 & 1.22 & 0.134 & $3.87 \times 10^{-18}$ \\
\hline-1.02000 & 1.81 & 0.198 & $5.72 \times 10^{-18}$ \\
\hline-1.01000 & 3.57 & 0.391 & $1.12 \times 10^{-17}$ \\
\hline-1.00900 & 3.96 & 0.434 & $1.25 \times 10^{-17}$ \\
\hline-1.00800 & 4.45 & 0.488 & $1.40 \times 10^{-17}$ \\
\hline-1.00700 & 5.08 & 0.557 & $1.60 \times 10^{-17}$ \\
\hline-1.00600 & 5.92 & 0.649 & $1.86 \times 10^{-17}$ \\
\hline-1.00500 & 7.09 & 0.777 & $2.24 \times 10^{-17}$ \\
\hline-1.00400 & 8.86 & 0.970 & $2.79 \times 10^{-17}$ \\
\hline-1.00300 & 11.7 & 1.29 & $3.72 \times 10^{-17}$ \\
\hline-1.00200 & 17.6 & 1.93 & $5.57 \times 10^{-17}$ \\
\hline-1.00100 & 35.2 & 3.86 & $1.11 \times 10^{-16}$ \\
\hline-1.00090 & 39.2 & 4.29 & $1.23 \times 10^{-16}$ \\
\hline-1.00080 & 44.0 & 4.83 & $1.39 \times 10^{-16}$ \\
\hline-1.00070 & 50.3 & 5.52 & $1.59 \times 10^{-16}$ \\
\hline-1.00060 & 58.7 & 6.44 & $1.85 \times 10^{-16}$ \\
\hline-1.00050 & 70.5 & 7.72 & $2.22 \times 10^{-16}$ \\
\hline-1.00040 & 88.1 & 9.65 & $2.78 \times 10^{-16}$ \\
\hline-1.00030 & 117 & 12.8 & $3.70 \times 10^{-16}$ \\
\hline-1.00020 & 176 & 19.3 & $5.56 \times 10^{-16}$ \\
\hline-1.00010 & 352 & 38.6 & $1.11 \times 10^{-15}$ \\
\hline-1.00009 & 391 & 42.9 & $1.23 \times 10^{-15}$ \\
\hline-1.00008 & 440 & 48.2 & $1.39 \times 10^{-15}$ \\
\hline-1.00007 & 503 & 55.1 & $1.58 \times 10^{-15}$ \\
\hline-1.00006 & 587 & 64.3 & $1.85 \times 10^{-15}$ \\
\hline-1.00005 & 704 & 77.2 & $2.22 \times 10^{-15}$ \\
\hline-1.00004 & 880 & 96.5 & $2.77 \times 10^{-15}$ \\
\hline-1.00003 & 1170 & 128 & $3.70 \times 10^{-15}$ \\
\hline-1.00002 & 1760 & 193 & $5.55 \times 10^{-15}$ \\
\hline-1.00001 & 3520 & 386 & $1.11 \times 10^{-14}$ \\
\hline
\end{tabular}

Table 6: Values of $\left(t_{\text {rip }}-t_{\text {caus }}\right)$ for $-1.10000 \leq \omega \leq-1.00001$ 


\section{Appendix B}

\section{Constraint on $\rho_{C}$ from causality}

There exists a lower bound on the value of $\rho_{C}$ coming from the causal disconnection condition for smaller bound systems such as the hydrogen atom and nucleon. We study here the lower bound on $\rho_{C}$ calculating $\left(t_{T}-\right.$ $\left.t_{\text {caus }}\right)$ numerically as a function of $w$ and $\rho_{C}$ for each bound system with a size $\lesssim 10^{-10} \mathrm{~m}$.

Imposing the condition $t_{T}>t_{\text {caus }}^{\mathrm{H}, \mathrm{N}}$ where $H, N$ denote the hydrogen atom and nucleon, respectively, we may obtain physical constraints on $\eta$ and $w$ from the following inequality:

$$
\begin{aligned}
\left(t_{T}-t_{\text {caus }}\right)^{\mathrm{H}, \mathrm{N}} & =\frac{|1+3 w|\left(L_{\mathrm{H}, \mathrm{N}} / c\right)}{3|1+w|}\left[1-\frac{3.12 \times 10^{11.95} / L_{\mathrm{H}, \mathrm{N}}}{\sqrt{\eta}|1+3 w|}\right] \\
& \geq 0,
\end{aligned}
$$

which follows from Eqs.(26) and (41). In Fig 3 we show the constraints on the model parameters $\eta$ and $w$, coming from the causal disconnection condition (42). Here we have taken $L_{\mathrm{H}}=0.5 \times 10^{-10} \mathrm{~m}, L_{\mathrm{N}}=10^{-15} \mathrm{~m}$, and [5] $c=2.9979 \times 10^{8} \mathrm{~m} \mathrm{~s}^{-1}, \mathrm{yr}=3.1556 \times 10^{7} \mathrm{~s}$. From this figure, we find a lower bound on the value of $\eta$, or equivalently on the critical density $\rho_{C}$,

$$
\eta \gtrsim 10^{54} \leftrightarrow \rho_{C}>\left(10^{9} \mathrm{GeV}\right)^{4},
$$

where we have calculated $\rho_{C}=\left(1.44 \times 10^{x / 4-4.5} \mathrm{GeV}\right)^{4}$ with $x=\log _{10} \eta$.

If we extend a similar study to a bound PPP system, for which we set a scale $L_{\mathrm{PPP}} \simeq 10^{-33} \mathrm{~m}$, slightly above the Planck scale, we find a stronger lower bound on $\eta$ (see the solid line illustrated in Fig. 3i),

$$
\eta \gtrsim 10^{90} \leftrightarrow \rho_{C} \gtrsim\left(10^{18} \mathrm{GeV}\right)^{4} .
$$

It is interesting to note that the result on these lower bounds is fairly insensitive to the choice of $w$ within the range of interest. 


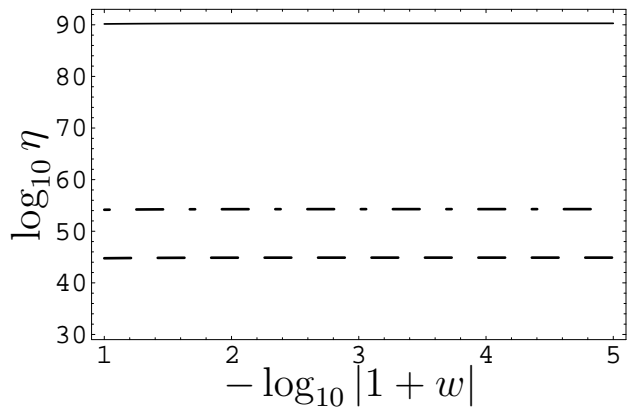

Figure 3: The lower bound on $\eta$ coming from the causal disconnection conditions, $t_{T}>$ $t_{\text {caus }}^{\mathrm{H}}\left(\right.$ dashed line), $t_{T}>t_{\text {caus }}^{\mathrm{N}}$ (dashed-dotted line), $t_{T}>t_{\text {caus }}^{\mathrm{PPP}}$ (solid line), for $10^{29} \leq \eta \leq$ $10^{93}$ and $-1.10000 \leq w \leq-1.00001$. The regions below these three lines are forbidden by causality. 


\section{Appendix $\mathrm{C}$}

\section{Comes back empty condition}

At $t=t_{T}$ we require that at deflation immediately prior to turnaround of the cyclic universe the causal patch comes back empty which demands that the deflation factor $f$ satisfies $f^{-3} \gtrsim 10^{102}$ in order to solve the entropy problem, since the present entropy is at least $10^{102}[8,9]$.

This requirement can be met by imposing that one causal patch be less than a size of the smallest bound systems, $L_{p}\left(t_{T}\right)$

$$
\frac{r_{H}\left(t_{T}\right)}{N_{\mathrm{cp}}} \leq L_{p}\left(t_{T}\right),
$$

where the subscript $p$ denotes a bound system whose size $L_{0}$ at present lies in the range $10^{-33} \mathrm{~m} \leq L_{0} \leq 10^{-15} \mathrm{~m}$. Noting that

$$
\begin{aligned}
r_{H}\left(t_{T}\right) & =r_{H_{0}} a\left(t_{T}\right), \\
L_{p}\left(t_{T}\right) & =L_{p}\left(t_{0}\right) \frac{a\left(t_{T}\right)}{a\left(t_{\text {unbound }}\right)},
\end{aligned}
$$

we can rewrite the condition (45) as

$$
a\left(t_{\text {unbound }}\right) \leq N_{\mathrm{cp}} \frac{L_{p}\left(t_{0}\right)}{r_{H_{0}}} .
$$

Looking at Eqs.(28), (31) and (37), we see that the left hand side of Eq.(48) can be reexpressed as

$$
\left[\frac{\sqrt{2|1+3 w|}}{4 \pi} P_{p}\left(H_{0} \sqrt{\Omega_{\Lambda}^{0}}\right)\right]^{\frac{2}{-3|1+w|}} \leq N_{\mathrm{cp}} \frac{L_{p}\left(t_{0}\right)}{r_{H_{0}}},
$$

where period $P_{p}$, associated with a certain smaller bound system $p$ we are concerned with, can be expressed as $P_{p} \simeq L_{p}\left(t_{0}\right) / c$.

Taking $\log _{10} \log _{10}$ of $N_{\mathrm{cp}}$ makes it easier to plot the inequality (49)). A plot of the $\left(w, \log _{10} \log _{10} N_{\mathrm{cp}}\right)$-plane varying the value of $L_{p}\left(t_{0}\right)$ in the range of interest, $10^{-33} \mathrm{~m} \leq L_{p}\left(t_{0}\right) \leq 10^{-15} \mathrm{~m}$ is shown in Figure 1, We have used [5] $r_{H_{0}}=1.232 \times 10^{26} \mathrm{~m}$ and $\Omega_{\Lambda}^{0}=0.76$. 


\section{References}

[1] L. Baum and P. H. Frampton, Phys. Rev. Lett. 98, 071301 (2007) hep-th/0610213.

[2] P.H. Frampton, Mod. Phys. Lett. A22, 2587 (2007). arXiv: 0705.2730.

[3] L. Baum and P.H. Frampton, Mod. Phys. Lett. A23, 36 (2008). hep-th/0703162.

[4] [Planck Collaboration], arXiv:astro-ph/0604069.

[5] W. M. Yao et al. [Particle Data Group], J. Phys. G 33, 1 (2006).

[6] D. N. Spergel et al. [WMAP Collaboration], Astrophys. J. Suppl. 170, 377 (2007) arXiv:astro-ph/0603449.

[7] R. R. Caldwell, M. Kamionkowski and N. N. Weinberg, Phys. Rev. Lett. 91, 071301 (2003) arXiv:astro-ph/0302506.

[8] P.H. Frampton and T.W. Kephart. arXiv:0711.0193.

[9] P.H. Frampton, S.D.H. Hsu, T.W. Kephart and D. Reed. arXiv:0801.1847.

[10] R.C. Tolman, Relativity, Thermodynamics and Cosmology. Oxford University Press (1934).

[11] P.J. Steinhardt and N. Turok, Science 296, 1436 (2002).

P.J. Steinhardt and N. Turok, Phys. Rev. D65, 126003 (2002). hep-th/0111098. L.A. Boyle, P.J. Steinhardt and N. Turok, Phys Rev. D70, 023504 (2004) hep-th/0403026.

P.J. Steinhardt and N. Turok, Science 312, 1180 (2006). astro-ph/0605173. 